\title{
SOME PROBLEMS IN THE STUDY OF VARIABLE STARS IN GLOBULAR CLUSTERS
}

\author{
B. V. KUKARKIN \\ Astronomical Council of the U.S.S.R. Academy of Sciences, Moscow, U.S.S.R.
}

\section{Introduction}

Three quarters of a century separates us from the beginning of systematic observations of variable stars in globular clusters. During the last $45 \mathrm{yr}$ the name of Mrs Prof. Dr H. B. Sawyer Hogg has always been in the astronomical literature. Her activity is closely connected with investigations of variable stars in globular clusters.

The complex dependences between the presence of variable stars of different types and the properties of the globular clusters were discovered long ago. For instance, the RR Lyr variables do not occur in clusters of high metallicity. Red variables on the contrary do not occur in clusters of very low metallicity. The presence and peculiarities of variable stars have been used for the classification of globular clusters (van Agt and Oosterhoff, 1959; Castellani et al., 1970).

Unfortunately, little attention has been paid to the problem of the frequency of variable stars in globular clusters. Extremely interesting is the problem of a spatial distribution of variable stars relative to stars of different sequences in globular clusters. Also very important is the correct investigation of the period changes of the RR Lyr and W Vir variables in globular clusters. Especially interesting is the study of the period changes in connection with the position of variable stars on the colourmagnitude diagrams.

\section{The Influence of Selection on the Frequency of Variable Stars in Globular Clusters}

The main selection factor among the samples of different types of variables in globular clusters is the probability of discovery. The probability depends upon the type of variability, the range of variation, the apparent density of stars in clusters, etc. Thus the probability of discovery of $R_{R_{c}}$-type stars is significantly smaller than for the $\mathrm{R}_{a b}$-type, while the probability of discovering eclipsing binaries is smaller than for the $\mathrm{RR}_{c}$ stars. The probability of finding $\mathrm{U}$ Geminorum type stars is still less. The usual method of observation of variable stars in globular clusters excludes the possibility of discovering $\mathrm{U}$ Gem stars and variables with very slow changes of brightness. The observers are usually interested in the RR Lyr variables and observe the globular clusters very intensively only during one or two months. The exposure time must be optimal for the RR Lyr variables. The probability of a $U$ Gem variable flaring in this short interval is very small. Moreover, the absolute magnitude of $U$ Gem variables at maximum is more than $2^{\mathrm{m}}-3^{\mathrm{m}}$ fainter than the RR Lyr stars at minimum. Therefore 
the $U$ Gem variables in globular clusters are usually fainter than the limiting magnitude of the plates.

The majority of the red variable stars in globular clusters are very poorly studied. Many of them have not been discovered. Only a few observatories have photographs suitable for the discovery and study of the slowly varying red stars. Due to the initiative of Mrs H. B. Sawyer Hogg the David Dunlap Observatory has a unique collection of plates for this purpose.

Insufficient allowance for selection leads to erroneous conclusions. For example, the globular cluster NGC 3201 was investigated on the basis of many Harvard Observatory plates (Wright, 1941). $56 \mathrm{RR}_{a b}$-type stars and only one $\mathrm{R}_{c}$-type star were studied and some stars were not solved. Further observation (Kukarkin, 1971a, b) brought the number of $R R_{c}$ variables to ten!

It is usually supposed that eclipsing binaries are rare in globular clusters. Actually the population of globular clusters does not correspond to the population of the majority of eclipsing binaries in our Galaxy or the Magellanic Clouds, but it is known that in globular clusters there exist stars of the U Gem-type and Novae, and in the extremely old open clusters there are W UMa-type stars. All these stars are much fainter than the RR Lyr variables. Unfortunately no one has systematically searched for variable stars in this magnitude interval in globular clusters.

It is clear that customary ideas on the frequency of variable stars of different types will be changed after accurate consideration of selection.

Thus the concept of frequency demands a more accurate definition. The frequency must be determined not by the absolute number of objects, but by the ratio of this number to the number related with the richness of the stellar system studied (e.g. the absolute magnitude $M$, the number of stars brighter than a set absolute magnitude, etc.).

The problem of the frequency of variable stars and other objects was considered some years ago (Kukarkin, 1968).

The frequency of given types of objects in a given stellar system is the ratio of the absolute number of objects to the total number of stars or to some value proportional to this number. The influence of selection and life-time must be considered.

\section{The Frequency of RR Lyr-, W Vir-, and RV Tau-Type Stars}

In the globular cluster $\omega$ Cen 139 RR Lyr-, $6 \mathrm{~W}$ Vir- and one RV Tau-type stars are known. In the globular cluster NGC 5053 only 10 RR Lyr stars and no W Virginis stars are known. According to the absolute number of variable stars $\omega$ Cen is extremely rich. But according to the richness of these two clusters this conclusion is erroneous. The absolute magnitude $M_{\mathrm{V}}$ of $\omega$ Cen is $-10^{\mathrm{m}} \cdot 2$ and of NGC 5053 only $-6^{\mathrm{m}} \cdot 1$ (Kukarkin and Russev, 1972). We assume that the general number of stars is proportional to the luminosity. We then find that $\omega$ Cen is 40 times richer than NGC 5053 so that it is necessary to reduce the number of variables in $\omega$ Cen by the factor 40 . We obtain $139 / 40 \simeq 3-4$ and $7 / 40 \simeq 0$. Consequently the frequency of RR Lyr stars in NGC 5053 is $3-4$ times greater than in $\omega$ Cen! 
In Table I the frequencies of RR Lyr stars are given for well observed globular clusters with reliable absolute integral magnitudes. In the first column is given the designation of cluster according to NGC Catalogue; in the second - the observed

TABLE I

The frequencies of RR Lyrae variables in globular clusters

\begin{tabular}{|c|c|c|c|c|c|}
\hline NGC & $n$ & $M_{\mathrm{V}}$ & $N$ & $I M$ & \\
\hline 104 & 2 & -8.93 & 0.5 & 0.56 & 4 \\
\hline 362 & 12 & -7.09 & 17 & 0.44 & 3 \\
\hline 2419 & 25 & -8.30 & 12 & 0.34 & 1 \\
\hline 3201 & 83 & -6.67 & 178 & 0.41 & 3 \\
\hline 4147 & 16 & -5.91 & 69 & 0.33 & 3 \\
\hline 4590 & 37 & $-6.9:$ & 64 & 0.22 & 2 \\
\hline 4833 & 11 & -7.47 & 11 & 0.31 & 3 \\
\hline 5024 & 33 & -8.54 & 12 & 0.30 & 4 \\
\hline 5053 & 10 & -5.91 & 43 & 0.25 & 3 \\
\hline 5139 & 137 & -10.19 & 11 & 0.35 & 5 \\
\hline 5272 & 200 & -8.29 & 97 & 0.38 & 5 \\
\hline 5466 & 20 & -6.53 & 49 & 0.26 & 4 \\
\hline 5824 & 25 & -7.61 & 22 & 0.29 & 2 \\
\hline 5897 & 5 & -7.11 & 7 & 0.26 & 1 \\
\hline 5904 & 92 & -8.24 & 46 & 0.39 & 5 \\
\hline 6121 & 40 & -6.24 & 127 & 0.45 & 4 \\
\hline 6171 & 22 & -6.27 & 69 & 0.50 & 5 \\
\hline 6205 & 2 & -8.05 & 1 & 0.34 & 4 \\
\hline 6218 & 0 & -7.15 & 0 & 0.35 & 4 \\
\hline 6229 & 20 & -7.26 & 25 & 0.42 & 3 \\
\hline 6254 & 0 & -7.26 & 0 & 0.36 & 4 \\
\hline 6266 & 81 & -8.69 & 26 & 0.45 & 2 \\
\hline 6333 & 11 & -7.76 & 9 & 0.32 & 1 \\
\hline 6341 & 13 & -8.01 & 8 & 0.25 & 4 \\
\hline 6352 & 0 & -5.31 & 0 & 0.65 & 1 \\
\hline 6362 & 23 & -6.18 & 78 & 0.43 & 3 \\
\hline 6397 & 0 & -6.32 & 0 & 0.33 & 4 \\
\hline 6402 & 62 & -8.79 & 19 & 0.42 & 4 \\
\hline 6426 & 12 & -5.63 & 67 & 0.33: & 1 \\
\hline 6626 & 7 & -7.99 & 4.5 & 0.47 & 1 \\
\hline 6637 & 0 & -7.84 & 0 & 0.66 & 2 \\
\hline 6656 & 17 & -8.17 & 9 & 0.32 & 4 \\
\hline 6712 & 9 & -6.81 & 17 & 0.51 & 3 \\
\hline 6715 & 54 & -8.61 & 19 & 0.43 & 3 \\
\hline 6723 & 19 & -7.38 & 21 & 0.51 & 3 \\
\hline 6752 & 0 & -7.35 & 0 & 0.35 & 1 \\
\hline 6779 & 0 & -7.10 & 0 & 0.31 & 4 \\
\hline 6809 & 6 & $-7.6:$ & 5: & 0.32 & 1 \\
\hline 6838 & 0 & -4.90 & 0 & 0.59 & 4 \\
\hline 6934 & 44 & -6.91 & 76 & 0.39 & 3 \\
\hline 6981 & 33 & -6.70 & 69 & 0.38 & 4 \\
\hline 7006 & 60 & -7.27 & 74 & 0.37 & 3 \\
\hline 7078 & 78 & -8.84 & 23 & 0.25 & 5 \\
\hline 7089 & 17 & -8.92 & 5 & 0.30 & 4 \\
\hline 7099 & 3: & -6.98 & 5: & 0.26 & 1 \\
\hline 7492 & 3 & -4.89 & 30 & 0.30 & 1 \\
\hline
\end{tabular}


number $n$ of RR Lyr stars; in the third - the absolute magnitude of clusters $M_{\mathbf{v}}$; in the fourth - the number $N$ of RR Lyr stars reduced to $M_{\mathrm{V}}=-7^{\mathrm{m}} .5$; in the fifth - the index of metallicity $I M$ and in the sixth - the estimate of reliability (5 - very good, 1 - very poor).

It is clearly seen that there are three areas of frequency of RR Lyr stars in globular clusters.

(I) In all the clusters with $I M \geqslant 0.57$ the RR Lyr stars are completely absent.

(II) In the clusters with $0.57>I M>0.32$ the number of RR Lyr stars increase with decreasing values of $I M$. The clusters NGC 3201, 6121 and 5272 have very high frequencies of RR Lyr stars. (The cluster IC 4499 is probably also extremely rich but unfortunately the type of variable star in this cluster is unknown and the value of $M_{\mathrm{V}}$ is very unreliable). The values of $I M$ in the second area are overlapped by the values in the third area, but both are very well separated (see Figure 1).

(III) Some clusters with $I M<0.37$ have no RR Lyr stars (NGC 6218, 6254, 6397 etc.). With the decreasing of $I M$ the number of RR Lyr stars increases.

The globular clusters which fall in the second area of Figure 1 show a preferential concentration of $\mathrm{RR}_{a b}$ variables, particularly those of the short-period group; clusters with longer-perıod $\mathbf{R R}_{a b}$ stars tend to lie in the third area, as do those with $\mathbf{R R}_{c}$ variables. Clusters with W Vir stars are present in both the second and third areas but absent from the first. Clusters with red variables are present in all three areas, but those with Mira stars are found only in the first area.

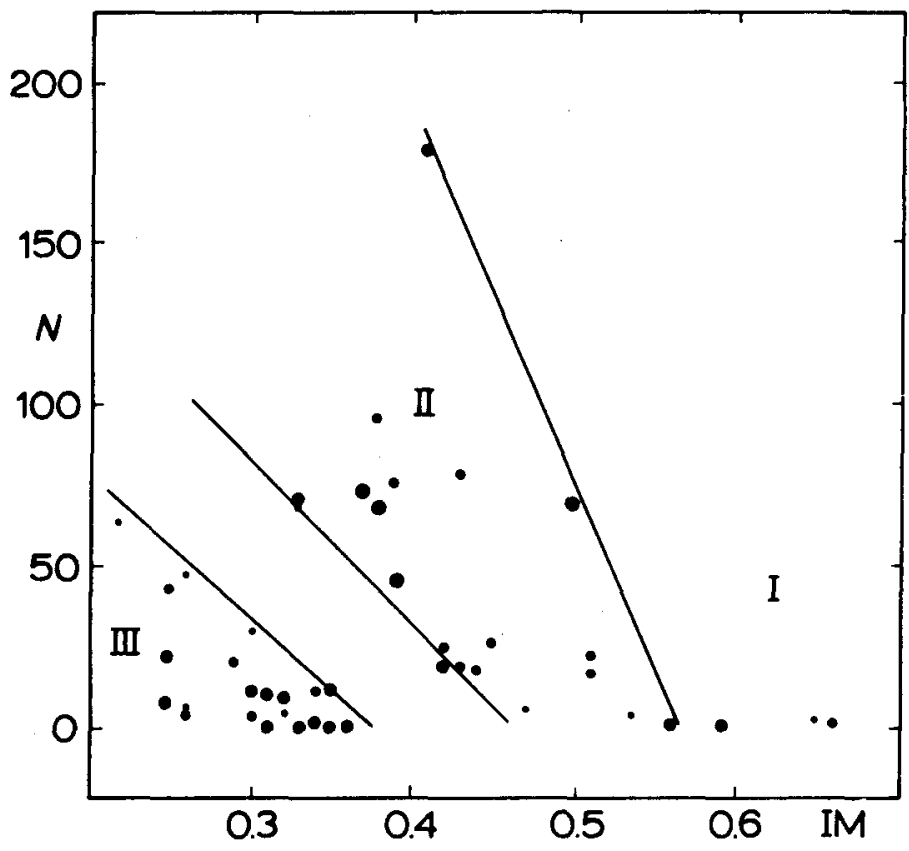

Fig. 1. The frequency of RR Lyrae variables as a function of the index of metallicity of the globular clusters. 
It is clear that the two-dimensional $N-I M$ relation, while requiring interpretation, is insufficient for describing the properties of globular clusters.

The following clusters have a very low frequency of RR Lyr stars: NGC 6397, $6218,6779,6254$. Perhaps the high frequency of RR Lyr variables is connected with the index of metallicity between 0.4 and 0.5 . The preliminary diagram of frequency of the RR Lyrae stars as a function of metallicity of the globular clusters is given in Figure 1.

The true frequencies of the RR Lyr stars have already been used for the classification of globular clusters (Mironov, 1973), but the problem deserves a thorough study.

TABLE II

W Vir-RV Tau variables and integrated properties of globular clusters

\begin{tabular}{lllll}
\hline NGC & $n$ & $M_{\mathrm{v}}$ & $N$ & $I M$ \\
\hline 5024 & 1 & -8.54 & 0.3 & 0.30 \\
5139 & 7 & -10.22 & 0.6 & 0.35 \\
5272 & 1 & -8.32 & 0.5 & 0.38 \\
5904 & 2 & -8.28 & 1.0 & 0.39 \\
6093 & 1 & -8.22 & 0.5 & 0.36 \\
6205 & 3 & -8.04 & 1.8 & 0.34 \\
6218 & 1 & -7.19 & 1.3 & 0.35 \\
6254 & 2 & -7.24 & 2.5 & 0.36 \\
6402 & 5 & -8.75 & 1.6 & 0.42 \\
6656 & 1 & -8.13 & 0.6 & 0.32 \\
6779 & 1 & -7.05 & 1.5 & 0.31 \\
7078 & 1 & -8.84 & 0.3 & 0.25 \\
7089 & 4 & -8.94 & 0.9 & 0.30 \\
\hline
\end{tabular}

In Table II the frequencies of W Vir plus RV Tau stars are given. It is necessary to remember the very small number of W Vir and RV Tau stars and the possibility of large fluctuations. But it is very probable that in the well studied clusters practically all the W Vir and RV Tau stars have been discovered. The structure of Table II is the same as Table $\mathrm{I}$.

The following clusters have a high frequency of W Vir and RV Tau variables: NGC 6254, 6205, 6402 and 6779.

It was recently shown that a high frequency of $\mathrm{W}$ Vir and RV Tau stars is connected with a high helium abundance (Mironov, 1973), but this conclusion requires further investigation.

The recent start on systematic observations of W Vir and RV Tau stars in globular clusters (Demers, 1971: Kukarkin and Rastorguev, 1972) is very welcome.

\section{The Frequency of Red Variable Stars}

The red variable stars (excluding Mira-type stars) are encountered more frequently in globular clusters than W Vir and RV Tau stars. A special list of red and similar 
variables in globular clusters was compiled by Stothers (1963). For some red variables the spectra and radial velocities were obtained by Joy (1949), but the photometric study of these stars is unsatisfactory. The principal reason for this is the very short time intervals during which most observations are obtained.

The great difficulty in the photometry of red variables is the unacceptability of the usual $U B V$ system. This unacceptability is caused by the very strong TiO absorption bands, especially in the $V$ region. Therefore the position of the red stars on the $V-(B-V)$ diagrams does not show the true position on the luminosity-temperature diagram. The search for good substitutes for the $U B V$ system is extremely significant (see Eggen, 1968, 1969; Lloyd Evans, 1971; Lockwood and Wing, 1971; Russev, 1972). It is obvious that further observations must be made without the use of the $V$ region; observations in the $B$ region are preferable. Therefore the observations in regions similar to $B$ may be very useful in the study of the variability of the red stars.

More than $15 \mathrm{yr}$ ago it was noted that the instability of red giants in globular clusters increases with their luminosity (Walker, 1955), an effect recently confirmed by Russev (1971). Figure 2 demonstrates the advantage of the photometric region $B$ as compared

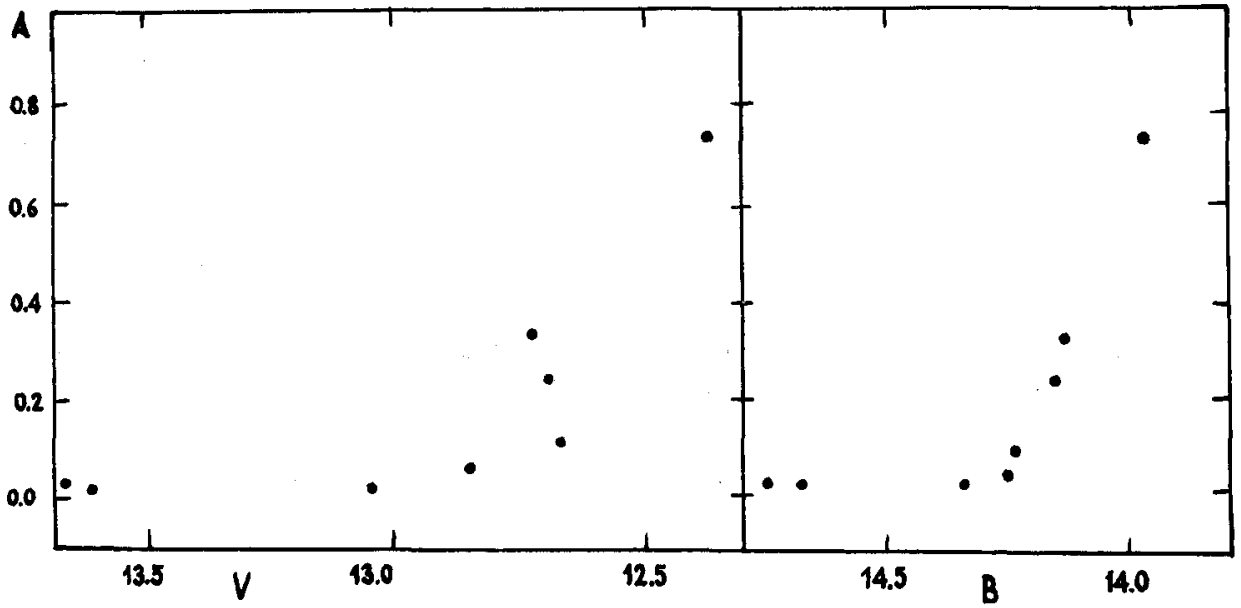

Fig. 2. The increase of instability with luminosity in the photometric region $V$ (left) and $B$ (right). The advantage of the $B$ region is evident.

with the $V$ region. The left part of the figure gives the 'magnitude $V$-Amplitude' relation (Russev, 1971). On the right part of the figure the $V$ magnitudes are replaced by the $B$ magnitudes. When the colour-magnitude diagram is expressed in a photometric system better than the $V,(B-V)$ one, all the red variables in globular clusters fall to the extreme right end of the giant branch. It is possible that all these stars are at the turning-point of evolution, near the helium flash.

The presence of red variables in such different globular clusters as 47 Tuc, M71, M13 and $\omega$ Cen is very significant. It is very probable that in a suitable photometric system the red variables would be separated. This separation should be related to age and chemical composition. 
We now have in Moscow a 12-yr series of B observations of such different clusters as M56 (index of metallicity $I M=0.30)$ and $\mathrm{M} 71(I M=0.60)$.

In Table III the frequencies of the red variables are given. The structure of Table III is the same as in Table $\mathrm{I}$. The numbers of red variables are small and there is no certainty that all the red variables have been discovered.

TABLE III

Red variables and integrated properties of globular clusters

\begin{tabular}{lllll}
\hline NGC & $n$ & $M_{v}$ & $N$ & $I M$ \\
\hline 104 & 6 & -8.93 & 1.6 & 0.56 \\
5139 & 10 & -10.22 & 0.9 & 0.35 \\
5272 & 3 & -8.32 & 1.4 & 0.38 \\
6121 & 2 & -6.20 & 6.6 & 0.45 \\
6205 & 2 & -8.04 & 1.8 & 0.34 \\
6656 & 3 & -8.13 & 1.7 & 0.32 \\
6779 & 4 & -7.05 & 6.1 & 0.31 \\
6838 & 2 & -4.91 & 21.7 & 0.59 \\
\hline
\end{tabular}

The new data on red variable stars are given by Lloyd Evans and Menzies in this book (pp. 151-163).

\section{The Frequency of Mira-Type Stars}

The membership of the three Mira-type stars in the globular cluster 47 Tuc is certain. It is also very probable that there are two Mira-type stars in the cluster NGC 6637 (Catchpole et al., 1970). The index of metallicity $I M$ of 47 Tuc is 0.57 , of NGC 6637 0.62 , which makes it likely that the presence of Mira-type stars is typical of high metallicity clusters.

There are some globular clusters with one or two Mira-type stars in their neighbourhoods. For some clusters (e.g. NGC 6093, 6171, 6656) it is clear that the Mira-type stars are field stars, but we do not know this for others. It is desirable that the radial velocities of the possible Mira-type members of globular clusters be determined. Some new data are given in Feast review (see pp. 131-144).

\section{The Frequency of Eclipsing Binaries}

Some globular clusters (e.g. NGC 3201, $\omega$ Cen, 6838) have one eclipsing binary. There is no convincing evidence for their membership, however, although in some cases their apparent magnitude does not rule out membership. But it is too early to speak about the frequency of eclipsing binaries.

The problem of the U Gem stars and novae in globular clusters was considered recently (Kurochkin and Kukarkin, 1966; Kukarkin and Mironov, 1970). A reliable solution of the problem demands systematic searches for variable stars in the magnitude interval usually neglected by observers (from $2^{\mathrm{m}}$ to $8^{\mathrm{m}}$ fainter than RR Lyr stars). 
Arp has kindly given us some plates of the globular cluster M5 made with the 200-in. Palomar telescope (limiting magnitude about $21^{\mathrm{m}}-22^{\mathrm{m}}$ ). Unfortunately only one pair of plates was available for comparison. Kurochkin made a thorough comparison of this pair, but no eclipsing or other variables except $V 101$ were discovered. However, this is not unexpected because of the very small probability of discovering eclipsing binaries.

The comparison of two pairs of plates taken with the 40-in. Burakan Schmidt telescope (limiting magnitude about $19^{\mathrm{m}}-20^{\mathrm{m}}$ ) has revealed one faint variable in the

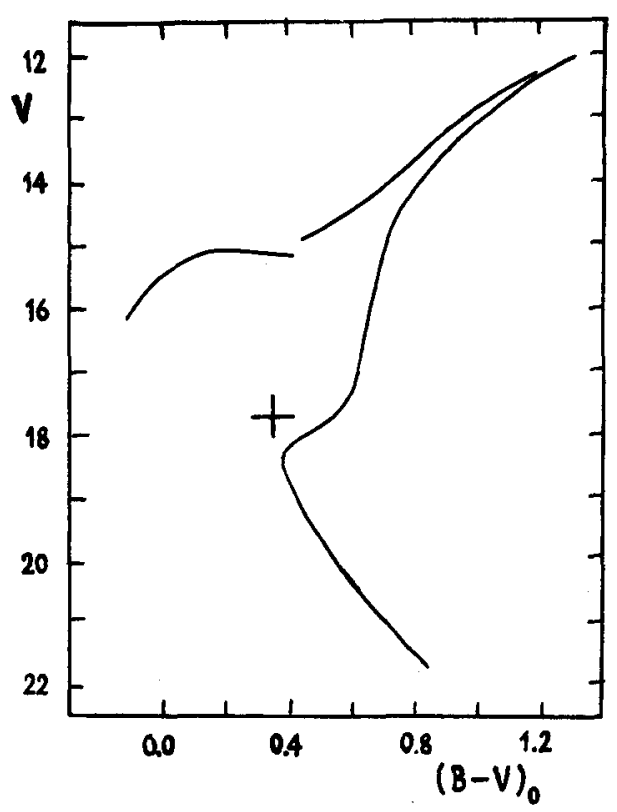

Fig. 3. The position on the colour-magnitude diagram of a new faint variable star in the globular cluster in M92.

neighbourhood of the globular cluster M92. This star is situated on the colourmagnitude diagram near the turn-off point of the main sequence. Its position is very similar to the position of W UMa variables (see Figure 3), but unfortunately the small number of plates does not allow the period or light curve to be determined.

The problem of eclipsing binaries in globular clusters requires very frequent observations.

Special attention must be paid to the search for eclipsing effects among RR Lyr stars. The discovery of such a star in the Ursa Minor dwarf galaxy (Kholopov, 1970, 1971 ) is very promising.

It is desirable to investigate thoroughly all pronounced fadings of RR Lyr stars in globular clusters, especially at light minimum. It is probable that such fadings have been accepted by observers as due to observational errors. 


\section{The Dissipation of Variable Stars and the Dynamical Evolution of Globular Clusters}

The dynamical history of any autonomic stellar system inevitably involves the problem of dissipation. The construction of colour-magnitude diagrams for comparatively outlying regions of globular clusters has shown that the number of cluster members is still high. Many papers by Kholopov have demonstrated the presence of very extended coronae (see e.g. Kholopov, 1968a).

The presence of appreciable members of RR Lyr stars in far outlying regions of some globular clusters was demonstrated very convincingly by Kurochkin (1961, 1962). These investigations were based on a search for variable stars up to $5^{\circ}$ around some globular clusters. Similar investigations are being carried out by the Italian astronomers (Rosino, 1970).

The RR Lyr stars are extremely useful for the study of dissipation, because their relationship with the given globular cluster is easy to establish. The probability that the apparent magnitude, period, shape of the light curve, etc. of the field RR Lyr variables will be similar to those in the given globular cluster is so slight that no doubt will arise.

In recent years many articles on the dynamic evolution of spherically symmetric stellar systems have been published (see for example King, 1962, 1966; Larson, 1970). Efficiently organised studies of variable stars in the extended surroundings of globular clusters must yield rich material for investigating the dynamical evolution of clusters. It is also possible that this material may give an independent method for determining the masses of globular clusters. Therefore it is most desirable to study many globular clusters of different masses and at different distances from the centre of our Galaxy. It would also be interesting to study this problem among globular clusters in the Magellanic Clouds.

\section{The Distribution of Stars of Different Types in Globular Clusters and the Problems of Stellar Evolution}

The problem of the distribution of stars of different types in clusters was studied long ago. But these investigations were not always correct. For instance Woolf's attempt to estimate the mass ratio of stars in the red and blue parts of the horizontal branch was hardly serious (Woolf, 1964), being based on a simple count of star numbers at different distances from the centre of the globular cluster M3.

The estimate of the mass ratio of RR Lyr and W Vir stars by Kukarkin and Voroshilov (1971) must be considered as preliminary. This estimate was based on the determination of the density gradient of the RR Lyr and W Vir stars. But the number of W Vir stars in globular clusters is small and it was therefore necessary to construct a synthetic globular cluster by reducing individual clusters to a common distance and radius. However, the established fact of the absence of W Vir stars and the presence of RR Lyr stars at great distances from the centres of clusters gives evidence of the larger masses of W Vir stars. 
In the past ten years more correct investigations have been carried out (see for example Kholopov, 1968a, b; Blaghikh and Castellani, 1971; Castellani et al. 1972). These papers showed that it is impossible to accept the equality of masses of all stars on the giant or horizontal branches. For the RR Lyr stars Kholopov has derived results contradicting the mass estimates for stars of other types. This contradiction is probably connected not only with the peculiarities of the RR Lyr stars themselves, but also with the space density of the stellar medium.

The correct study of the problem of space distribution of stars of different types in globular clusters is extremely important. The determination of the mass ratio of stars of different types must be the test of the numerous theoretical calculations (see for example Schwarzschild, 1970; Schwarzschild and Härm, 1970; Rood, 1970; Iben and Rood, 1970; Demarque and Geisler, 1971; Iben, 1972; Demarque and Mengel, 1972; etc.).

\section{The Variability of the Periods of Variable Stars}

In the twenties and thirties of our century it was customary to represent the deviations of the epochs of variable stars from the linear elements by second order equations. The coefficient of the second order term was accepted as the value describing the progressive change in the period. In this way it was established that the instability of the period of the classical Cepheids increases with the period itself (Florja and Kukarkin 1932). This same technique was applied to the RR Lyr stars in globular clusters (see for example Martin, 1938, 1942). The coefficient of the second order term was designated in many articles as $\beta$. Such an approach to the problem at that time is excusable because the second order equations represented the deviations of the epochs satisfactorily. But single variable stars show more complex deviations.

Numerous new observations and a considerable increase in the intervals covered by observations have shown that in reality the phenomenon is very complex. The periodicity of a pulsating star undergoes random fluctuations which are spontaneous variations having quite different origins. We often observe successive changes of period, in which it increases then decreases, and vice versa. In these cases the epochs can be represented very well by two or more systems of linear equations. This was clearly demonstrated as long ago as the mid-fifties by Parenago (1956).

The intervals of observation of the RR Lyr stars are short and reach at best $80 \mathrm{yr}$, yet it is clear that the observed changes of period must be considered as noise. This noise may in fact veil the slow evolutionary changes.

It is not impossible that a proper examination of the period-noise of many RR Lyr stars in some rich globular clusters by modern methods of mathematical statistics may lead to the discovery of the evolutionary changes of periods. But it is necessary to proceed with extreme caution. The level of noise varies among different types of RR Lyr stars in the same globular cluster. It is extremely large for the RR Lyr stars showing the Blazhko-effect, but there are other RR Lyr stars with quite constant periods over intervals of 70 or even $80 \mathrm{yr}$.

The quantity $\beta$ is also a certain measure of noise, but the approach to the problem 
must be completely revised. Especially important is the study of noise in connection with the peculiarities of the RR Lyr stars and their positions on the colour-magnitude diagram. Quite probably there exists a relationship between the noise-level and the peculiarities of the RR Lyr stars.

It is also very desirable to study the period changes of the W Vir- and RV Tau-type stars.

\section{The Conclusions}

In this short survey we are considering only some problems arising from the study of variable stars in globular clusters. We attempt to select those problems the solving of which can easily be put into practice. Here is a short list of these probiems :

(1) Closer investigation into the frequency of variable stars of different types in various globular clusters, taking selection effects into account.

(2) Comparing the frequencies of variable stars of different types with the peculiarities of globular clusters.

(3) The search for variable stars in those intervals of stellar magnitude in which a search has not yet been made.

(4) The study of the red variable stars in new photometric systems satisfactorily representing the bolometric magnitudes and temperatures.

(5) The search for and study of variable stars in the extended surroundings of globular clusters for the consequent investigation of the dissipation problem.

(6) The correct investigation of the distribution of different variable stars in various globular clusters.

(7) The study of fluctuations in the periods (noise) of variable stars with consideration of the positions of variables on the colour-magnitude diagram and the comparison with theories of stellar evolution.

\section{References}

Agt, S. van, and Oosterhoff, P. Th.: 1959, Ann. Sterrew. Leiden 21, 253.

Blaghikh, A. and Castellani, N.: 1971, Mem. Soc. Astron. Ital. 42, 23.

Castellani, N., Giannone, P., and Renzini, A.: 1970, Astrophys. Space Sci. 9, 418.

Castellani, N., D'Antona, F. A., and Natta, A.: 1972, Mem. Soc. Astron. Ital. 43, 167.

Catchpole, R. M., Feast, M. W., and Menzies, J. U.: 1970, Observatory 90, 63.

Demarque, P. R. and Geisler, J. E.: 1971, Astrophys. J. 164, 469.

Demarque, P. R. and Mengle, J. G.: 1972, Astrophys. J. 171, 583.

Demers, S.: 1971, Astron. J. 76, 916.

Eggen, O. J.: 1968, Astrophys. J. Suppl. 16, 49.

Eggen, O. J.: 1969, Astrophys. J. 158, 225.

Florja, N. F. and Kukarkin, B. V.: 1932, Z. Astrophys. 4, 247.

Iben, I.: 1972, Publ. Astron. Soc. Pacific 83, 697.

Iben, I. and Rood, R. T.: 1970, Astrophys. J. 161, 587.

Joy, A. H.: 1949, Astrophys. J. 110, 105.

Kholopov, P. N.: 1968a, Astron. Zh. 45, 768.

Kholopov, P. N.: 1968b, Astron. Zh. 45, 1235.

Kholopov, P. N.: 1970, Astron. Tsirk. No. 601.

Kholopov, P. N.: 1971, Perem. Zvezdy 18, 117.

King, I. R.: 1962, Astron. J. 67, 471.

King, I. R.: 1966, Astron. J. 71, 64. 
Kukarkin, B. V.: 1968, in Problems of Star Evolution and Variable Stars, Navka Press, Moscow, p. 120.

Kukarkin, B. V.: 1971a, Perem. Zvezdy 17, 610.

Kukarkin, B. V.: 1971b, Astron. Tsirk No. 637.

Kukarkin, B. V. and Mironov, A. V.: 1970, Astron. Zh.47, 1211.

Kukarkin, B. V. and Rastorguev, A. S.: 1972, Astron. Tsirk. No 707.

Kukarkin, B. V. and Russev, R. M.: 1972, Astron. Zh. 49, 121.

Kukarkin, B. V. and Voroshilov, Ju. V.: 1971, Astron. Zh. $48,1087$.

Kurochkin, N. E.: 1961, Perem. Zvezdy 13, 248.

Kurochkin, N. E.: 1962, Perem. Zvezdy 14, 196.

Kurochkin, N. E. and Kukarkin, B. V.: 1966, Astron. Zh. 43, 83.

Larson, R. B.: 1970, Monthly Notices Roy. Astron. Soc. 150, 93.

Lloyd Evans, T.: 1971, Private communication.

Lockwood, G. W.: 1971, Astrophys. J. 169, 63.

Martin, W. C.: 1938, Ann. Sterrew. Leiden 17, No. 2.

Martin, W. C.: 1942, Astrophys. J. 95, 314.

Mironov, A. V.: 1973, Astron. Zh. 50, 27

Parenago, P. : 1956, Perem. Zvezdy 11, 236.

Rood, R. T.: 1970, Astrophys. J. 161, 145.

Rosino, L.: 1970, Trans. IAU. XIVA, 292.

Russev, R. M.: 1971, Perem. Zvezdy 18, 171.

Russev, R. M.: 1972, Thesis.

Schwarzschild, M.: 1970, Quart. J. Roy. Astron. Soc.11, 12.

Schwarzschild, M. and Härm, R.: 1970, Astrophys. J.160, 341.

Stothers, R.: 1963, Astron. J. 68, 242.

Walker, M. F.: 1955, Astron. J. 60, 197

Woolf, N. J.: 1964, Astrophys. J.139. 1081.

Wright, F. W.: 1941, Harvard Bull. No. 915.

\section{DISCUSSION}

Wesselink: Why has Kukarkin not mentioned 47 Tuc as a cluster poor in RR Lyrae variables, although scaling it down (which you have to do) makes the number even smaller?

Feast: For some reason it is not in his paper.

Jones: Is not TiO observed only rarely in Globular Clusters?

Feast: It appears to be fairly common in related metalrich clusters (see my review tomorrow).

Schwarzschild: After listening to the broad reviews by Dr Hogg and Dr Kukarkin I am impressed and bewildered by the immense volume of the observational work in the field of pulsating variables in globular clusters which present tools permit us to do and which the impressive growth of results already achieved may suggest for the future. I wonder therefore whether the time has not come for us to distinguish sharply between further 'survey work' which might mainly aim at ascertaining that we are not missing a major type of cluster (in the sense of the essential astrophysical parameters) and on the other hand 'intensive work' on sample clusters - selected for observational accessibility -, one or two samples for each major cluster type. Such intensive work - as for example Dr Kukarkin's ninth point - would seem necessary to truly exploit the already fascinating observational data for theoretical deductions.

Wesselink: Concerning the matter of RR Lyrae's in the outskirts of clusters: I studied the field of $\omega$ Cen to $1^{\circ}, 2^{\circ}, 3^{\circ}$ away from centre and found RR Lyrae stars, but they are probably not members because they are fainter than the other RR Lyrae's.

Demarque: Regarding point \#9 raised by Prof. Kukarkin and commented upon by Prof. Schwarzschild, how reliably can one draw conclusions on the relative masses of different groups of stars, such as the RR Lyrae and W Virginis stars? Could anyone comment on the relative magnitudes of the evolutionary time-scales (about $10^{8} \mathrm{yr}$ between the beginning of the horizonal branch phase to the end of the asymptotic branch phase) and the relaxation times of the clusters? Assuming that differences in central concentration of different groups of stars can be established, should we expect to learn about the masses of the object observed, or rather about the masses of their progenitors if, for example, mass loss has occurred? 
Dickens: What are typical evolutionary times between horizontal branch and W Virginis phases? Relaxation times could be as short as $10^{8} \mathrm{yr}$ near the centres of some globular clusters, although for large clusters such as M3 they are an order of magnitude longer.

Wesselink: No $\delta$ Scuti stars were found on long exposure plates of $\omega$ Cen by the Leiden observers at the Radcliffe Observatory.

Graham: Are there not special observational problems in detecting variable stars of faint absolute magnitudes in globular clusters?

Wesselink: The problem is of course more difficult than the discovery of RR Lyrae's for two reasons:

(1) The fainter brightness requires longer exposures.

(2) The absolutely fainter stars exhibit changes which are more rapid.

Nevertheless the sensitivity of presently existing equipment is good enough to find such stars if they exist.

Rosino: It would be very difficult to find flare stars in globular clusters, since these stars have an extremely rapid variation, the time from minimum to maximum and back to minimum being sometimes as short as $5-10 \mathrm{~min}$ - so if the exposure time is of the order of $30^{\mathrm{m}}$ these stars will certainly be lost. 